\title{
Internal pressure driven finite element model of a single pulmonary acinus
}

\author{
James Campbel1 ${ }^{*}$, Salman Siddiqui ${ }^{2}$, Simon Gill ${ }^{1}$, and Alkiviadis Tsamis $^{1,3}$ \\ ${ }^{1}$ School of Engineering, University of Leicester, University Road, Leicester, LE1 7RH, UK \\ ${ }^{2}$ NIHR Leicester Biomedical Research Unit, Glenfield Hospital, Leicester, LE3 9QP, UK \\ ${ }^{3}$ Department of Mechanical Engineering, University of Western Macedonia, Bakola \& Sialvera \\ Street, Kozani, 50132, Greece
}

\begin{abstract}
A computer simulated, poroelastic, hyperelastic model was developed to replicate the pressure-volume response of a single pulmonary acinus ( $15^{\text {th }}$ branch of the respiratory tree and daughter branches) with air flow at its core. An internal pressure driven approach was taken upon a small spherical geometry $\left(99.2 \mathrm{~mm}^{3}\right.$ in volume) representing this small segment of lung parenchyma. A reference porcine tracheal pressure at tidal breathing was adjusted from $1471 \mathrm{~Pa}$ to $998 \mathrm{~Pa}$ to accommodate for pressure drop, and the pressure of $998 \mathrm{~Pa}$ was applied to the model for parametric analysis of its pressure-volume characteristics. In targeting a proportional tidal volume change of approximately $15 \%$ while also inducing a pressure-volume hysteresis, material parameters of Young's modulus of $4 \mathrm{kPa}$, Poisson's ratio of 0.4 , and a permeability of $5 \times 10^{-5} \mathrm{~cm}^{3} \mathrm{~s}^{-1} \mathrm{~cm}^{-2}$ were identified as suitable. The energy loss over a single pressure-volume cycle for a pulmonary acinus was found to be $6.3 \times 10^{-6} \mathrm{~J}$. This model was qualitatively compared to the pressure-volume relationship of the original porcine data source, and then with experimental findings of the material parameters for lung parenchyma in medical literature, demonstrating same-order agreement.
\end{abstract}

\section{Introduction}

The human lungs provide a huge point of interest in the field of clinical and computational medicine, not least with the increasing incidence of lung pathologies worldwide due to factors such as air pollution. The acinar airway - the site of gas exchange in the lungs and the vast majority of their surface area - is vitally important in the physiological function of the human body. Its enormous liquid-lined surface area, and hence site of surface tension, is also responsible for the phenomenon of hysteresis: observed as the inspiration of the breathing cycle requiring a greater pressure gradient to 'reopen' the lungs for any given volume compared to the expiratory part of the cycle [1].

A limitation of literature data on the smallest parts of the lung tissue (lung parenchyma) is that of the difficulty in probing airways so small on a living, breathing patient to determine their material properties. Studies could be undertaken on excised, post-humous parenchymal tissue, but this brings with it two particular issues; there may be structural changes of the

* Corresponding author: jc702@le.ac.uk 
tissue once removed from the body (i.e. due to deflation), and observations will not be drawn from said tissue while it is performing its living physiological behaviour.

One may observe that in existing work on modelling the lungs, the upper respiratory tree may be replicated (and can be done with good accuracy using imagery such as chest computed tomography (CT) scans) but due to the limitations in resolution of these image sources, the distal regions of the lungs are simplified - essentially a 'balloons on sticks' approach [2]. Furthermore, there may be a difficulty in the computational demand required to model a full set of lungs with the inclusion of the smallest airways.

In this study, a reverse approach is followed: building a poroelastic hyperelastic geometry that can represent a pulmonary acinus - a distal part of the respiratory tree, from the $15^{\text {th }}$ generation downwards - to be exposed to variable pressure waveforms. The resulting volume changes can then be extrapolated for comparison with pressure-volume relationships of the lung as a whole from reference data. In pursuing this method, one can aim to estimate the material parameters of living pulmonary acini within the body, which may in turn be used to tailor and simplify existing models of the lungs. Additionally, by investigating the permeability of this porous medium, one may seek to generate a pressure-volume hysteresis analogous to that seen physiologically albeit underpinned by flow dynamics as opposed to surface tension forces.

\section{Method}

\subsection{Constructing the geometry}

The point of origin on the respiratory tree for the small-scale geometry is that of the transitional bronchiole, the $15^{\text {th }}$ generation branch wherein the conducting airway (consisting of larger, more rigid cylindrical airway lumens that facilitate laminar air flow) becomes the diffuse airway (where alveoli budding emerges and the gas exchange between the airway and the vascular perfusion occurs). In Weibel et al. [3], a 'pulmonary acinus' is defined as being the network of alveolar airways that share a connection to a first order transitional bronchiole - with this acting as the 'stem' for the acinus (an image of this unit of lung tissue can be seen in Figure 1, right). One may assume that air flow will take place within this individual structure but not interconnect with any adjacent pulmonary acini, so any model must allow air flow from the transitional bronchiole substitute, but not via the external boundary.

To recreate this, a simple, perfectly spherical geometry was constructed in 2Daxisymmetrical space (for ease of computation) to represent this small portion of lung parenchyma from an individual transitional bronchiole downwards to the end of its alveolus bed. To calculate the volume of the geometry required, the total lung volume during the midpoint of tidal breathing (3.25 litres in a physiologically healthy $70 \mathrm{~kg}$ man [4] was divided by $2^{15}$ (or 32765 ) as the structure of the respiratory tree is that of divisions into two at each branch generation. There should therefore be approximately 33000 individual transitional bronchioles in a set of human lungs, each supplying air flow to a calculated $9.92 \times 10^{-5}$ litres (or $99.2 \mathrm{~mm}^{3}$ ) volume of parenchymal tissue.

The total radius of this geometry in the finite element package COMSOL Multiphysics $\mathrm{v} 5.5$, to produce this volume of $99.2 \mathrm{~mm}^{3}$ in $3 \mathrm{D}$ space, was $2.87 \mathrm{~mm}$. At the core of the geometry, the transitional bronchiole was replicated by constructing a smaller sphere of radius $0.25 \mathrm{~mm}$ (found through the inverse logarithmic relationship between branch and lumen size [5]). In Figure 1, the final geometry cross section can be seen (Figure 1, left) alongside an image of an actual pulmonary acinus (Figure 1, right). 

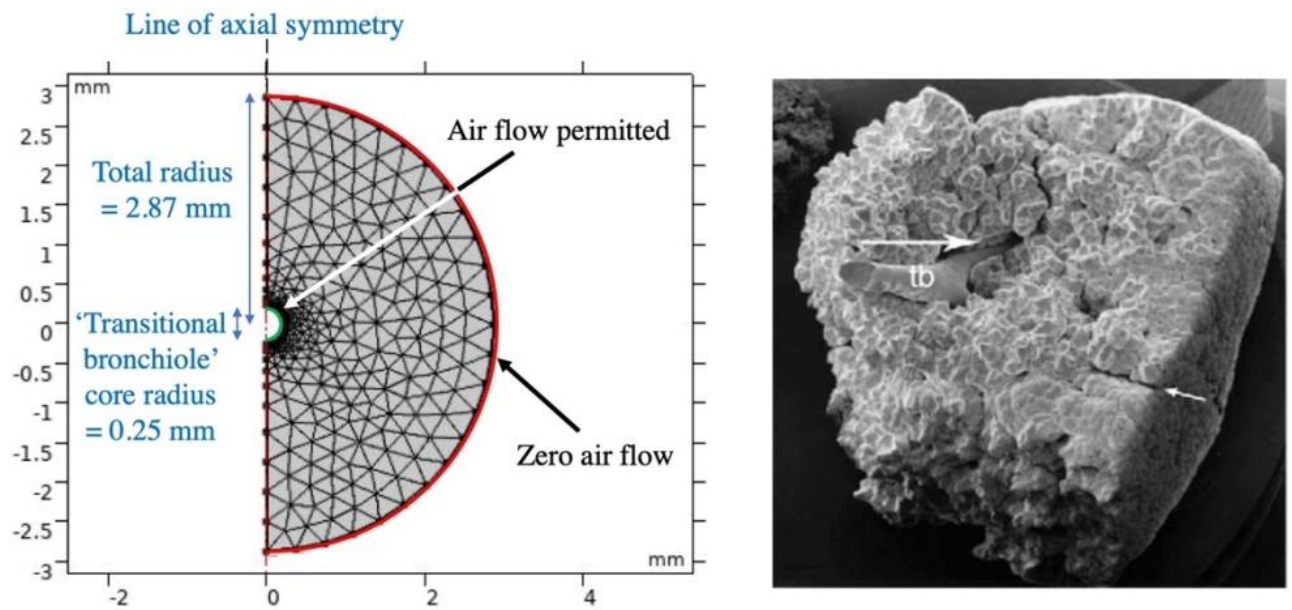

Fig. 1. Left: 2D cross section of geometry simulating pulmonary acinus. Right: scanning electron microscope image of human pulmonary acinus obtained through silicon casting, with the transitional bronchiole (tb) labelled [6].

\subsection{Selection of pressure waveform}

Because the alveoli are directly connected to the atmosphere via the respiratory tree, it is assumed that their internal pressure (the intra-alveolar pressure) and by extension the internal pressure of the entire acinar airway will be equalised with atmospheric pressure at rest. Here, the atmospheric pressure at rest is considered to be the central pressure of tidal breathing, with positive and negative deviations promoting volume changes in either direction over the course of a pressure cycle. Also of consideration is that the rate of tidal breathing lies between 10 and 20 breaths per minute [7]. Therefore, for the purposes of this model, a time-dependent pressure waveform of the form displayed in Equation 1 was used to control the amplitude of the wave $(A)$ and give a periodicity of 4.2 seconds, a rate of 14.3 'breaths' per minute.

$$
p(t)=A \sin (0.5 \pi t)
$$

To find a suitable value of $A$ for Equation 1, data from a study involving the mechanical ventilation of anaesthetised porcine specimens [9] was used (with pig lungs being similar to human lungs in size and anatomy [8]). That study provided a value of the peak tracheal pressure relative to the atmosphere and a corresponding volumetric change from the application of this pressure [9]. From this, a peak tracheal pressure gradient of approximately $15 \mathrm{cmH}_{2} \mathrm{O}$, or $1471 \mathrm{~Pa}$, was identified.

\subsection{Calculating pressure drop from trachea to transitional bronchiole}

The above-mentioned raw tracheal pressure of 1471 Pa required modification for use in the pulmonary acinus model. Any pressure gradient present at the trachea will be of a greater magnitude than that at an individual transitional bronchiole due to an inevitable pressure loss as air passes through the respiratory tree.

To quantify this, an adapted version of Poiseuille's law was used to estimate the resistance to air flow over each generation of the respiratory tree (assuming equal branching and a conservation of cross-sectional area between branches). Adding the resistances as a parallel system for each branch gave a pressure drop of $473 \mathrm{~Pa}$, or $32 \%$, between the $1^{\text {st }}$ and $15^{\text {th }}$ 
generations to $998 \mathrm{~Pa}$. For the sinusoidal pressure waveform $p(t)$ in Equation 1, $A=998 / 2$ to give a total maximum pressure differential of $998 \mathrm{~Pa}$.

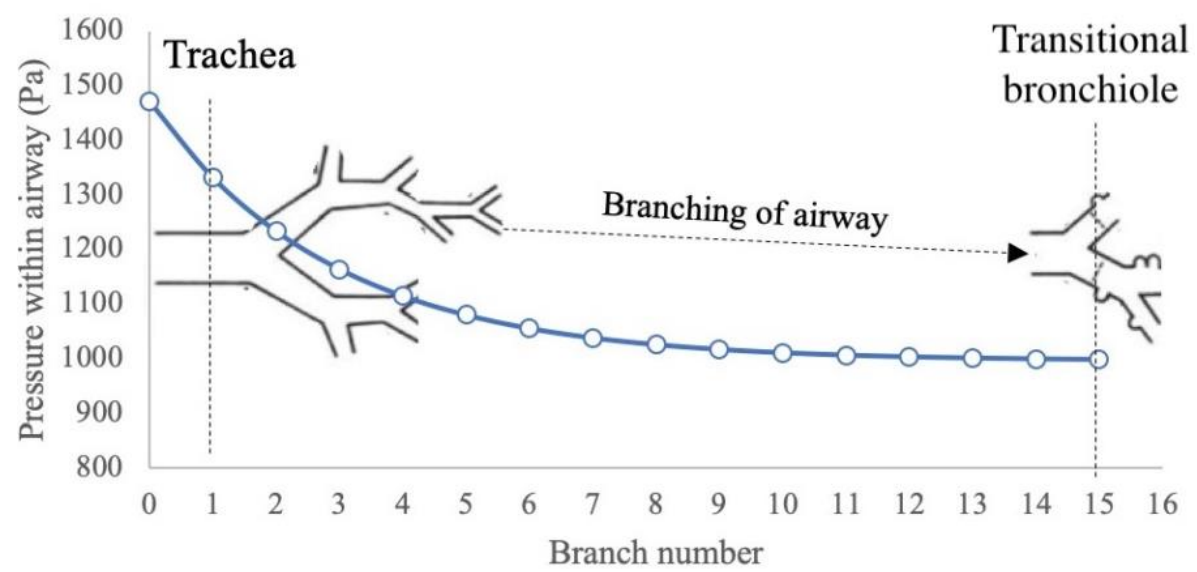

Fig. 2. Mathematically calculated pressure drop over branches of the conducting airway, illustrated using figures from Weibel [5, modified].

\subsection{Internal pressure driven model}

Using the geometry seen in Figure 1 (left), the pressure waveform $p(t)$ was applied at the boundary of the 'transitional bronchiole' core. Time-dependent studies of 20 seconds duration with a time step of 0.01 seconds were used with the model probed to determine the change in volume. This allowed for a direct comparison between the internal pressure and volume at any given time.

The poroelastic nature of the model denotes a porous medium wherein fluid flow is permitted and interacts with the deformation of the solid as a whole. 'Permeability' within this study is therefore the ability for the fluid to flow throughout the geometry. Parametric analysis with a constant, implausibly high value of permeability $\left(1 \mathrm{~cm}^{3} \mathrm{~s}^{-1} \mathrm{~cm}^{-2}\right.$, to prevent any hysteresis effects or permeability-based limitations on volume change) demonstrated that a Young's modulus $(E)$ and Poisson's ratio $(v)$ of $4 \mathrm{kPa}$ and 0.4 , respectively, gave the most appropriate deviations in volume from the geometry's original state. The volume deviations replicated the expected change across tidal breathing, which was an approximate $15 \%$ difference between peak and trough volumes $(500 \mathrm{~mL}$ [10] compared to the aforementioned 3.25 litres lung capacity midway through tidal breathing).

\section{Results}

\subsection{Parametric analysis of permeability}

Permeabilities ranging from $10^{-2}$ to $10^{-6} \mathrm{~cm}^{3} \mathrm{~s}^{-1} \mathrm{~cm}^{-2}$ were then used while maintaining a Young's modulus of $4 \mathrm{kPa}$ and Poisson's ratio of 0.4 . At lower permeabilities, this model tended to require up to three cycles to stabilise its pressure-volume relationship, meaning for the purposes of analysis and comparison the final pressure cycles for each were used. A selection of these data sets are plotted on Figure 3. 


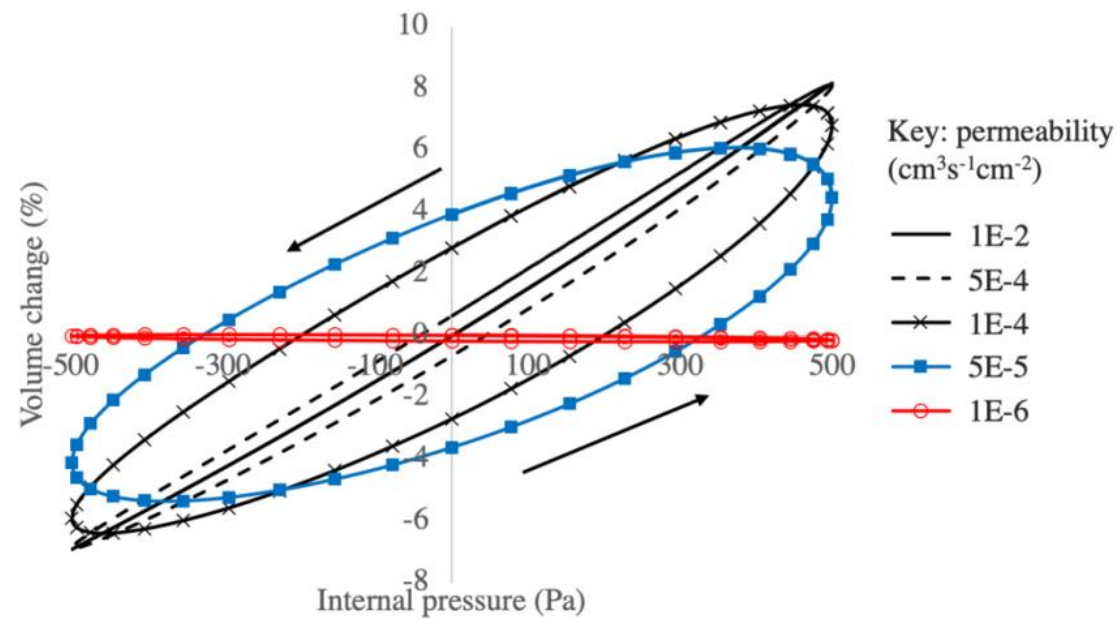

Fig. 3. Pressure-volume relationship for the internal pressure driven model $(E=4 \mathrm{kPa}, v=0.4)$ with a variable permeability (directionality of loop is indicated with arrows).

\subsection{Energy loss and maximum volume range}

To compare the aforementioned permeabilities quantitatively, their maximum change in volume (minimum to maximum values) was plotted as a percentage of the original geometry volume alongside the energy loss value for a single loop of the pressure-volume cycle. The latter value offers a single quantifiable measurement of the size of the pressure-volume hysteresis loop present for each permeability.

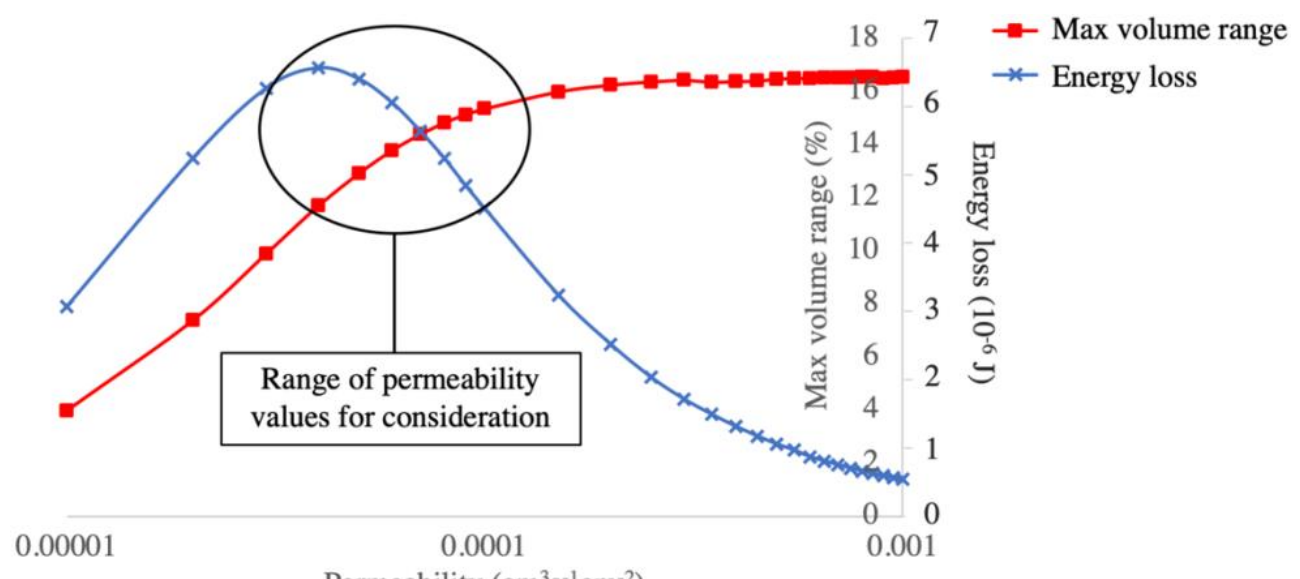

Permeability $\left(\mathrm{cm}^{3} \mathrm{~s}^{-1} \mathrm{~cm}^{-2}\right)$

Fig. 4. The maximum volume range (as a percentage of the original geometry volume) and energy loss per cycle for the internal pressure model $(E=4 \mathrm{kPa}, v=0.4)$ with a variable permeability.

From the data shown in Figures 3 and 4, and balancing the requirement of a sufficient volumetric change under the tidal breathing pressure conditions with the emergence of a significant pressure-volume hysteresis, the material parameters in Table 1 are suggested as suitable for a simulated pulmonary acinus. 
Table 1. Selected material parameters to represent pulmonary acinus.

\begin{tabular}{|c|c|c|}
\hline Parameter & Value & Units \\
\hline Young's modulus & 4 & $\mathrm{kPa}$ \\
\hline Poisson's ratio & 0.4 & - \\
\hline Permeability & $5 \times 10^{-5}$ & $\mathrm{~cm}^{3} \mathrm{~s}^{-1} \mathrm{~cm}^{-2}$ \\
\hline
\end{tabular}

\subsection{Comparison with reference data}

A direct comparison of the pressure-volume data set for the selected material parameters in Table 1 with the literature data for an intubated porcine specimen can be seen in Figure 5. The volume and pressure axis for each is adjusted to be proportional to the greatest volume change and greatest pressure, respectively. The energy loss of a single pressure-volume loop was found to be $6.3 \times 10^{-6} \mathrm{~J}$ for the internal pressure pulmonary acinus model $(E=4 \mathrm{kPa}, v=$ 0.4 ), and estimated at approximately $0.202 \mathrm{~J}$ for the porcine lung data.

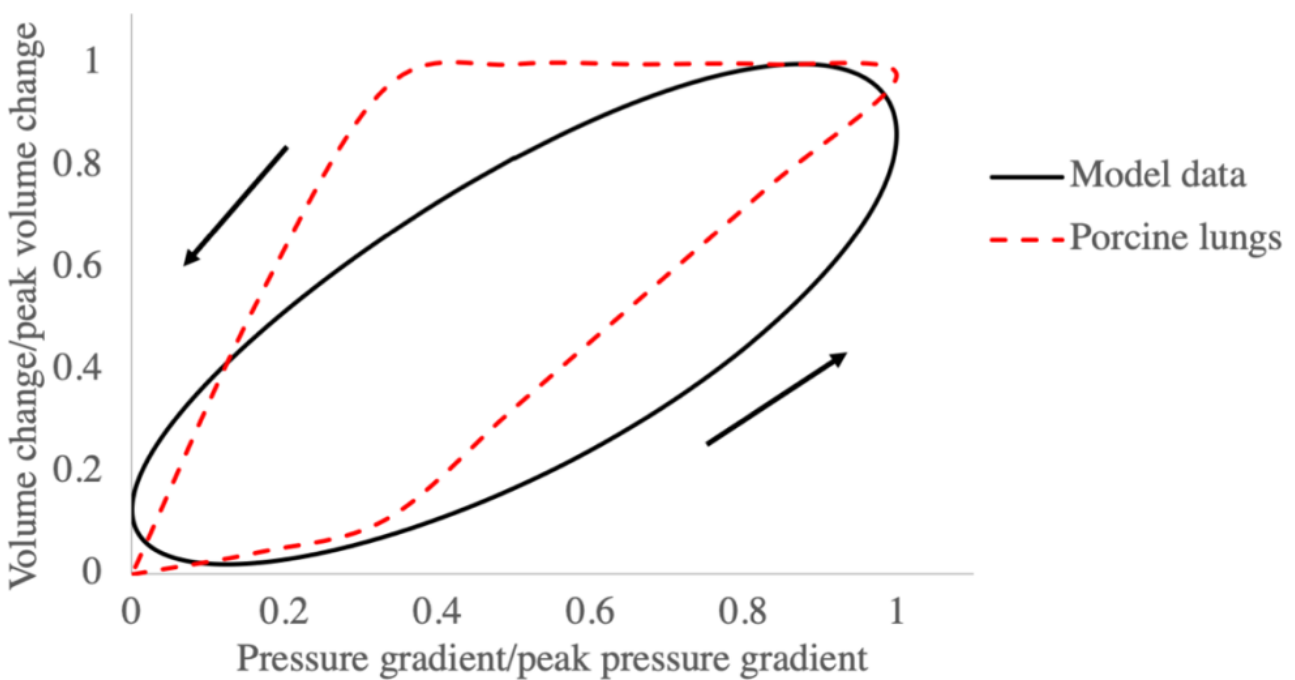

Fig. 5. Comparison of pressure-volume data for internal pressure model $(E=4 \mathrm{kPa}, v=0.4)$ with the reference data for a porcine specimen during ventilated tidal breathing [9] (with directionality of loop indicated).

\section{Discussion}

Qualitative comparison of the study's model with the reference porcine pressure-volume data shows a comparable relationship. Of particular note is that the internal pressure model shares the same, desired, directionality of cycle - though rather than being the result of surface tension forces seen physiologically, the model's hysteresis arises from a sufficiently low permeability causing flow during 'inspiration' to require a higher pressure gradient than that during 'expiration'. The profile of the two data sets does vary, however, but this likely reflects 
the simplicity of the small spherical model developed in this study (giving a simple, smooth pressure volume curve) compared to the more complex scenario in the pig experiment, which would be influenced by the thoracic wall, adjacent anatomical structures, gravity, etc.

Quantitatively, the estimated energy loss per cycle of $0.202 \mathrm{~J}$ for the porcine data should be directly compared to the calculated total lung energy loss for the study data (i.e. for $2^{15}$ pulmonary acini constituting the lungs as opposed to one), which equates to $0.206 \mathrm{~J}$. Though these are in close agreement, when one considers that the pigs in question were $26 \pm 2 \mathrm{~kg}$ in mass [9], and the model was designed with a $70 \mathrm{~kg}$ man in mind, one would expect a higher computational value for the internal pressure model's energy loss per cycle - and perhaps not one this close to the porcine data. However, this is likely a result of the adapted (and therefore lower) pressure amplitude in the model compared to the higher pressure in the reference data set, and the subsequent calculation of energy loss. Regardless, their same order of magnitude shows promise for the simulated tissue.

Given that the material parameters identified as suitable in Table 1 were found entirely through the relationship between the volume and internal pressure of the simulation, one may look to physical reference data to establish whether they agree with one-another. In a study using different methods of lung tissue measurements, porcine lungs were found to have a Young's modulus of 2.55-3.02 kPa using mechanical micro-indentation, and $3.68 \pm 0.06 \mathrm{kPa}$ using ultrasound [11]. These values, particularly the latter, are not only of the same magnitude as the Young's modulus used in this model $(4 \mathrm{kPa})$, but lie close to its value indicating its validity. One experimental study looking into the Poisson's ratio of lung parenchyma found it to be 0.424 [12], again, close to the value used in the model (0.4), though one would expect its value to lie between 0.4 and 0.5 regardless as a high-water-content organic material.

The true structure of a pulmonary acinus will be far more complex in reality than the homogeneous medium used in this study, although the model in itself is intended to offer a simple method of replicating the parenchymal behaviour. Unlike the Young's modulus and Poisson's ratio, there is therefore no equivalent 'permeability' value to be compared to for validation with real-world measurements. Instead, the selected permeability value of this simulated poroelastic medium $5 \times 10^{-5} \mathrm{~cm}^{3} \mathrm{~s}^{-1} \mathrm{~cm}^{-2}$ (or of a similar order of magnitude) offers a simplified parameter that can be used as representative of the lung parenchyma collectively.

\section{Model limitations and future work}

The source of tracheal pressure, from porcine studies, will likely differ to a small extent from the equivalent human pressures given morphological differences between the two, such as a longer trachea and increased asymmetry of the lungs in pigs [8]. However, the source of this data also provided volume-pressure relationships that would be too invasive to find in a human study (but were of particular use in this study for comparison), and was therefore appropriate. There will likely be scope to tailor this model to a different tracheal pressure moving forward using human medical data.

While the model in this study was limited to the tidal breathing volumetric range of the lungs, it may now be adapted to introduce higher pressure gradients in the pursuit of replicating more extreme deformations of the lung: seeking to reach the upper and lower boundaries (proportionately, in the case of the pulmonary acinus) of the lung capacity in humans. Consequently, this may allow for the replication of lung function tests upon the simulated medium, as peak inspiratory values are often a key part of this clinically [13].

In its current iteration, the model is $2 \mathrm{D}$-axisymmetric in nature for simplicity - however, a subsequent fully $3 \mathrm{D}$ incarnation will allow for much greater flexibility in terms of geometry selection (for example, using more complex shapes than a sphere, or asymmetrical features such as moving the 'transitional bronchiole' from the core). Whether in its 2D-axisymmetric 
or a fully $3 \mathrm{D}$ form, this model, having focussed upon physiologically healthy values, may now be altered to attempt the replication of pathological states such as obstructive or restrictive lung disease.

\section{Conclusions}

Modelling of the lungs often focusses upon the higher, conducting, airways and indeed can neglect the smaller airways when assessing macroscopic lung function. Due to the limitations of imaging technology and the physical testing of excised tissue, these smaller, acinar airways may also be neglected in terms of understanding their true characteristics while being part of a living, breathing set of lungs.

The bottom-up approach in this study to replicate the pressure-volume behaviour of lung parenchyma, and by extension the entire lungs, appears to have been successful. For a simple, homogeneous, poroelastic hyperelastic medium model, the material parameters identified and shown in Table 1 give a pressure-volume relationship that appears to match existing experimental reference data for known (and adapted) physiological pressure conditions. In this work, the physical characteristics of the pulmonary acinus as a whole have been attributed to a simplified sphere of appropriate size, and the significance of this may be to offer a tailored set of material parameters to pre-existing lung models that focus more on the conducting airways of the respiratory tree. The implementation of these parameters into other models may also present a feedback loop wherein the medium of this project may be finessed further after being used in a larger system and results are gathered.

More work will be conducted upon the presented internal pressure pulmonary acinus model to improve its complexity and explore patterns of behaviour beyond tidal breathing, including pathological states. In doing this, one hopes to find new insight into the way small airways act while breathing, and how they may be simulated in a computationally simple fashion.

\section{References}

1. J.D. Escolar, A. Escolar, Histol. Histopathol. 19159 (2004)

2. B.H. Foy, D. Kay, Respir. Physiol. Neurobiol. 24061 (2017)

3. E. R. Weibel, Respir. Physiol. Neurobiol. 1482 (2005)

4. D. Chambers, C. Huang, G. Matthews, Basic Physiology for Anaesthetists ( $2^{\text {nd }}$ edition) 50 (2019)

5. E. R. Weibel, Swiss Med. Wkly. 139375 (2009)

6. B. Haefeli-Bleuer, E.R. Weibel, Anat. Rec. 220401 (1988)

7. S.R. Braun, Clinical Methods: The History, Physical, and Laboratory Examinations. Chapter 43: Respiratory Rate and Pattern (3 ${ }^{\text {rd }}$ edition) 226 (1990)

8. E.P. Judge, J.M.L. Hughes, J.J. Egan, M. Maguire, E.L. Molloy, S. O’Dea, Am. J. Respir. Cell. Mol. Biol. 51334 (2014)

9. S. Schumann, U. Gobel, J. Haberstroh, L. Vimlati, M. Schneider, M. LichtwarckAschoff, J. Guttmann, Minerva Med. 8019 (2014)

10. S. Hallett, F. Toro, J.V. Ashurst, Physiology, Tidal Volume [Online] StatPearls

[Internet] https://www.ncbi.nlm.nih.gov/books/NBK482502/ (2021)

11. S.R. Polio, A.N. Kundu, C.E. Dougan, N.P. Birch, D.E Aurian-Blajeni, J.D. Schiffman, A.J. Crosby, S.R Peyton, PLoS One 1310 (2018)

12. J.P. Butler, M. Nakamura, H. Sasaki, T. Sasaki, T. Takishima, Jap. J. Phys. 36, 91 (1986)

13. J.D. Johnson, W.M. Theurer, Am. Fam. Physician 85359 (2014) 